\title{
NEMATIC HOMOGENEOUS PHOTO ALIGNMENT BY POLYIMIDE EXPOSURE TO LINEARLY POLARIZED UV
}

\author{
MASAKI HASEGAWA AND YOICHI TAIRA \\ IBM Research, Tokyo Research Laboratory \\ 1623-14, Shimotsuruma, Yamato-shi, Kanagawa 242, JAPAN
}

\begin{abstract}
Exposure of positive-type polyimide (PI) to linearly polarized deep UV (DUV) light $(257 \mathrm{~nm})$ induced homogeneous alignment of nematic liquid crystals. The alignment state changed markedly at a particular energy of the UV light. Measurement of the UV absorption spectrum and retardation change generated in PI films by UV irradiation showed that anisotropic alignment was caused by a new mechanism, photodepolymerization of polyimide main chains parallel to the electric field of the DUV light.
\end{abstract}

\section{Introduction}

The technology used in manufacturing liquid crystal display panels has made remarkable progress. However, the liquid crystal alignment technology is still dependent on the rubbing process. Since this creates dust, clean alternative techniques are needed. Other techniques for establishing a wide viewing angle are also needed as alternatives to mono-domain alignment. One alternative approach is to use an optical process; for example, several photochemical alignment methods have already been reported. The alignment direction of a rubbed dye-doped polyimide changes when the material is illuminated with linearly polarized light [1]. The alignment can be modulated by inducing a photochromic reaction [2]. Photopolymerization of a photopolymer with linearly polarized light has been shown to induce uniaxial orientation of liquid crystals [3]. Uniaxial orientation by linearly polarized light has been reported [2,3]. In these studies, however, particular materials, such as photopolymer and azobenzen, were used for the alignment layers. In manufacturing, it is normal to use positive-type polyimide (PI), whose characteristics in liquid crystal displays are well known. We tried to use this type of PI as an alignment layer, and to develop a new optical alignment method. Foe this reason, we exposed PI to linearly polarized deep UV (DUV) light and observed the alignment of liquid crystals that was induced by this procedure. 


\section{Experiment}

We spin-coated a glass substrate with positive-type PI AL1254 (Japan Synthetic Rubber Co., Ltd., Japan), which was developed for an alignment layer of active matrix liquid crystal display panels. This is soluble in $\gamma$-butyrolactone. The substrate was baked at $180^{\circ} \mathrm{C}$ for one hour to vaporize the solvent. Figure 1(a) shows an experimental arrangement for irradiation of linearly polarized DUV. A DUV beam is generated by a BBO crystal as a second harmonic of a $514.5 \mathrm{~nm}$ beam from an $\mathrm{Ar}^{+}$laser. We exposed the PI to $10 \mathrm{~mW}$ linearly polarized $257 \mathrm{~nm}$ DUV light, and assembled a cell with both top and bottom plates made of this DUV-exposed PI-coated substrate. The spacing between the two plates was $12 \mu \mathrm{m}$. After assembly, the cell was filled with 5CB liquid crystals at room temperature in the nematic phase. Figure 1(b) shows an assembly of the cell.

(a) Configuration of UV irradiation

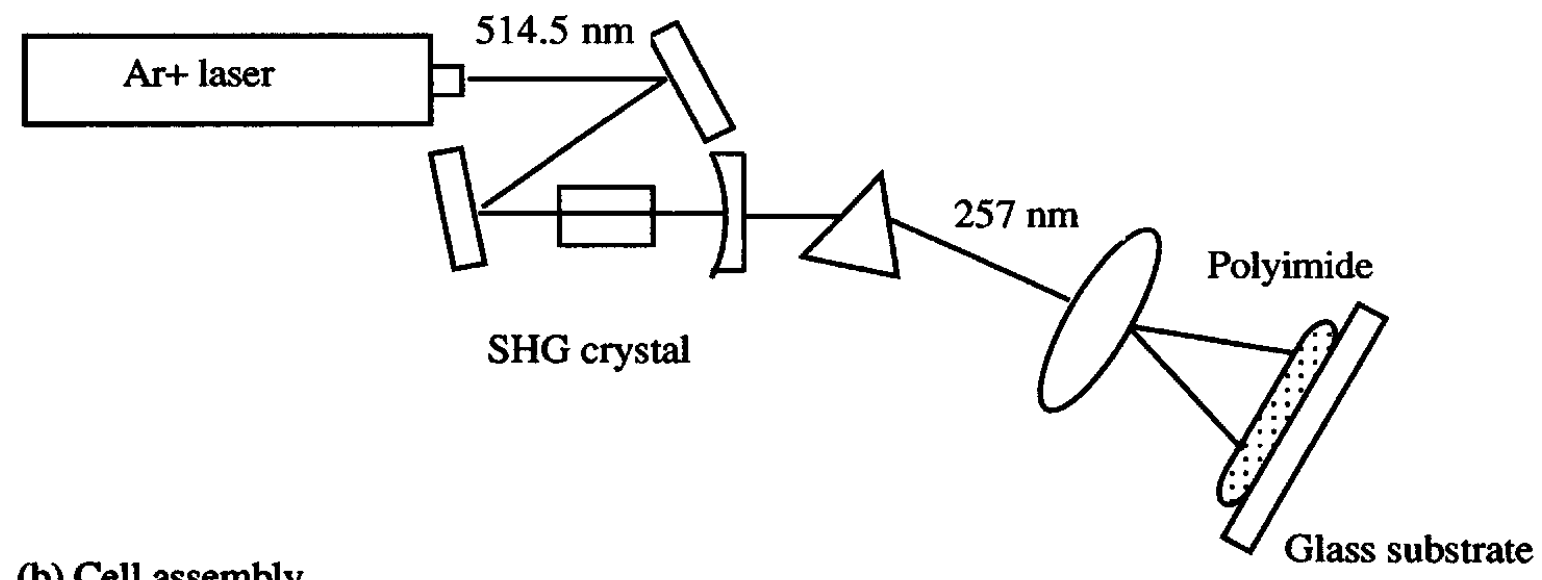

(b) Cell assembly

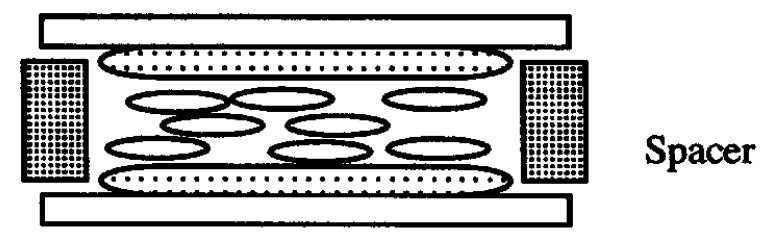

Fig. 1 Configuration of (a) DUV irradiation equipment and (b) a cell assembly 


\section{Results and discussion}

\section{Liquid crystal alignment}

We observed the alignment by using a polarization microscope. Figure 2 shows a photograph of the cell between crossed polarizers. The circle in the center of the photograph is the DUV-exposed PI, and the one in the upper part is the masked PI. The DUV-exposed PI induced a monodomain homogeneous alignment, while the masked PI showed only a flow alignment. To determine the alignment direction, we assembled a wedge cell. The alignment direction was perpendicular to the electric field polarization of the DUV.

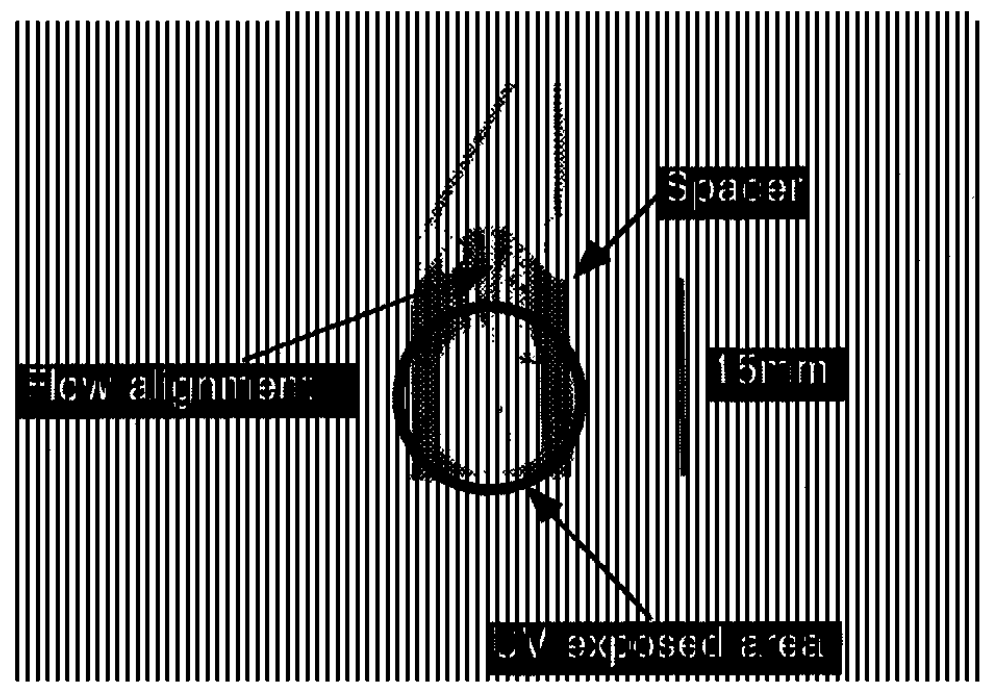

Fig. 2 Photograph of the cell betweeen crossed polarizers

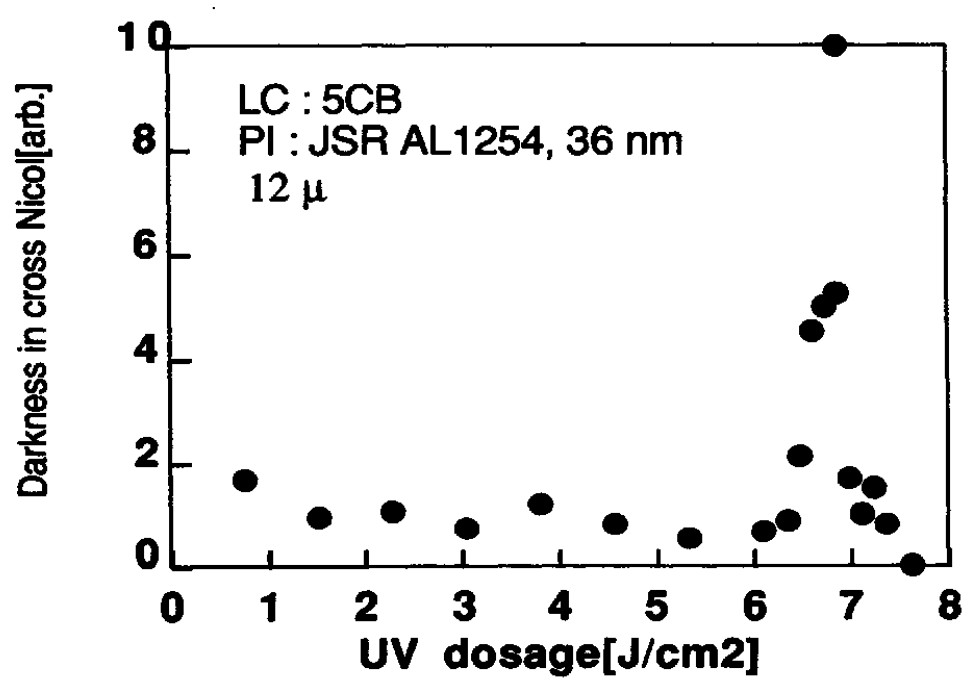

Fig. 3 Alignment state and UV energy to which the PI is exposed 
We changed the exposed energy by altering the exposure time. The alignment appeared at the lowest energy used in the experiment $\left(800 \mathrm{~mJ} / \mathrm{cm}^{2}\right)$, and disappeared at energies higher than $9 \mathrm{~J} / \mathrm{cm}^{2}$. To determine the alignment state, we measured the transmissivity of the cell between two crossed polarizers. Figure 3 shows the relation between the darkness of the cell and the exposed energy. The darkness was defined as $1 /$ transmissivity, and represents the alignment state. The darker the cell, the better the alignment. All of the measuring point alignments were monodomain and homogeneous, but the alignment state changed suddenly near $7 \mathrm{~J} / \mathrm{cm}^{2}$. At $7 \mathrm{~J} / \mathrm{cm}^{2}$, its state was as good as in rubbing alignment. Above $7 \mathrm{~J} / \mathrm{cm}^{2}$, however, the alignment became loose, and soon the monodomain alignment was not induced. To confirm the photochemistry of PI, we measured the following characteristics.

\section{Photochemical change in polyimide}

To determine the mechanisms of the alignment, we measured several changes in the characteristics of the PI resulting from exposure to DUV. The structure of the PI we used is shown in Figure 4. The UV absorption spectrum for the PI sample is shown in Figure 5. The upper curve shows the spectrum before UV irradiation, while the lower curve shows the spectrum after exposure to $257 \mathrm{~nm}$ DUV with an energy of $100 \mathrm{~J} / \mathrm{cm}^{2}$. The upper curve has three peaks, located at $200(P 1)$, 228 (P2), and $255 \mathrm{~nm}$ (P3). Although it is not clear by shown in Figure 5, a subtraction spectrum (Figure 6) shows that, in addition to these three decreased peaks, the lower curve has another broad peak, located at $286 \mathrm{~nm}(\mathrm{P} 4)$. The absorbance change in each peak is shown in Figure 7. The absorbance in P1 decreased markedly, while those of P2 and P3 decreased only slightly. P4 increased slightly, and saturated at $55 \mathrm{~J} / \mathrm{cm}^{2}$. To determine the cause of the reduced absorption, we measured the thickness of the PI film. Figure 8 shows the result. The thickness changed remarkably after exposure to DUV with an energy of $60 \mathrm{~J} / \mathrm{cm}^{2}$. However, the thickness change was not a cause of the absorption change, because the tendency of the absorption reduction is unrelated to that of the thickness change. We have not yet determined the molecular structure corresponding to each peak, but it is clear that $257 \mathrm{~nm}$ DUV destroys structures that have P1, P2,or P3 absorption peaks, and generates a new structure that has a $\mathrm{P} 4$ absorption peak.

We also measured the retardation change as a function of the DUV energy to which the PI is exposed. Figure 9 shows the retardation change in PI. It increased rapidly at the beginning of DUV irradiation, and saturated after irradiation of $10 \mathrm{~J} / \mathrm{cm}^{2}$. We measured this in two different conditions, to determine the effect of the oxygen: in the atmosphere, and while $\mathrm{N}_{2}$ gas was flowing over the 
surface of the film. There was no remarkable difference between the results in the two conditions. This means that oxygen does not affect this photochemistry. The increasing in the retardation provides evidence of anisotropic photochemical reactions.

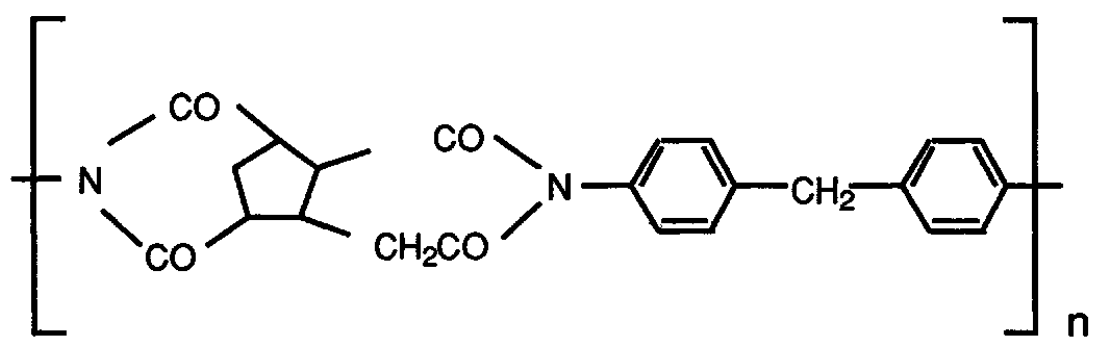

Fig. 4 Molecular structure of AL1254 polyimide

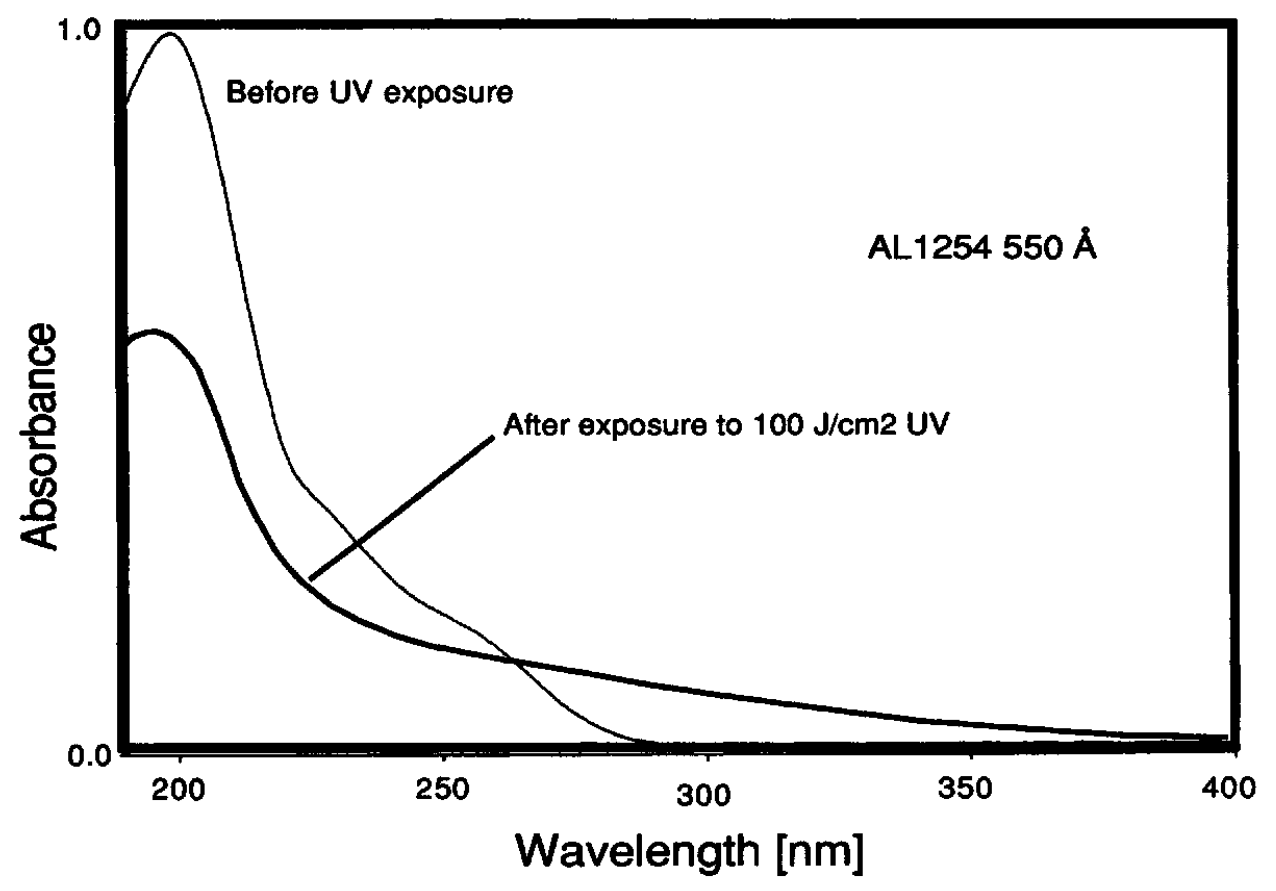

Fig. 5 UV absorption spectra before exposure to UV (thick line) and after exposure at an energy of $100 \mathrm{~J} / \mathrm{cm}^{2}$ (thin line) 


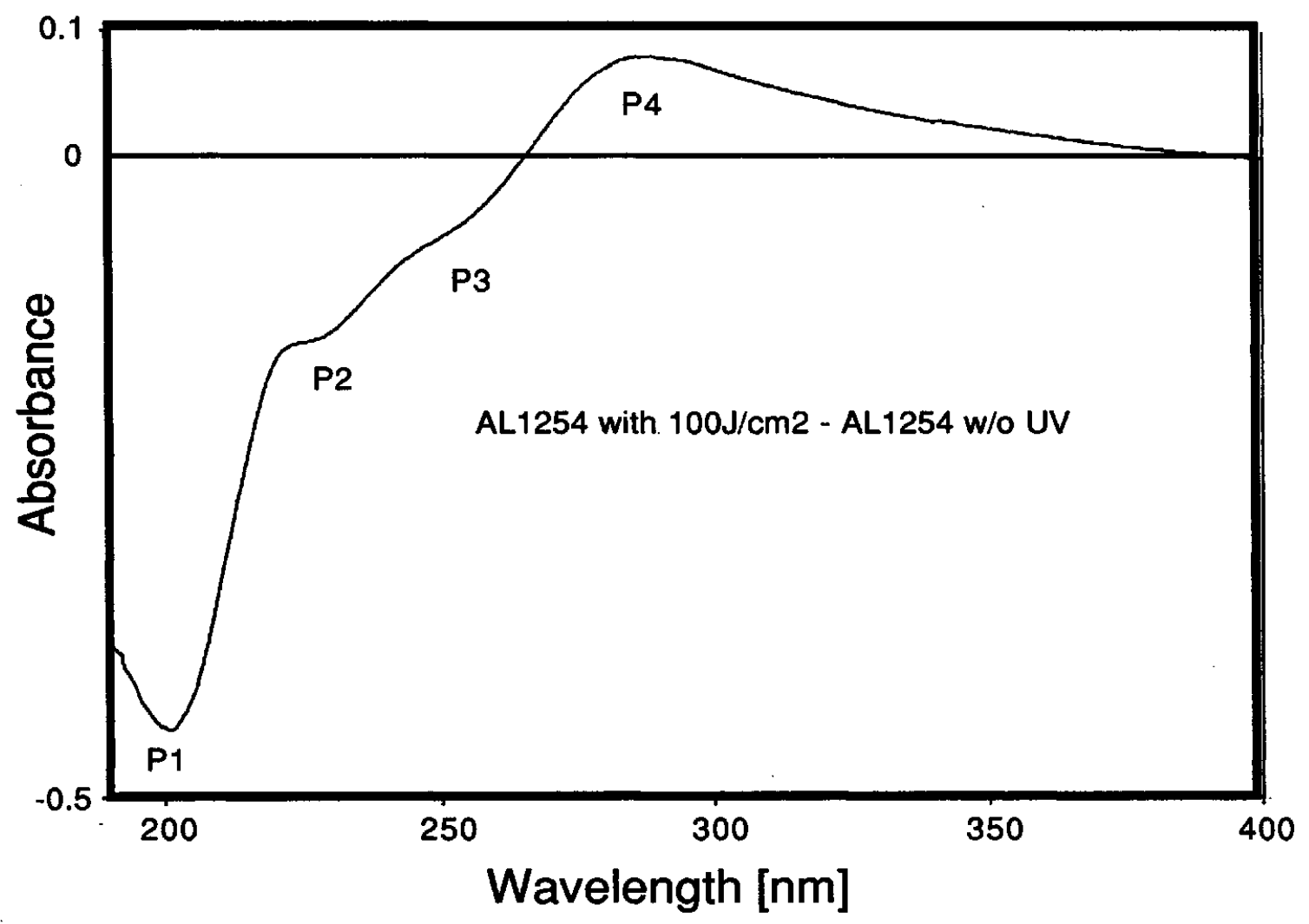

Fig. 6 Subtraction spectra before and after UV irradiation

\section{Alignment model}

Our experiments yielded three main results: the alignment direction is perpendicular to the electric vector of DUV, several UV absorption peaks of PI were decreased, and the retardation of PI was increased by DUV irradiation. The alignment direction, perpendicular to the electric field of DUV, shows that the alignment strength in that direction is greater than in other directions. The change in the alignment state shows that the anisotropy of PI is largest at DUV irradiation with an energy of $7 \mathrm{~J} / \mathrm{cm}^{2}$. When PI is irradiated with linearly polarized DUV, the main chain of PI parallel to the electric field of the DUV is broken more easily than chains in other directions, because the absorption of the PI parallel main chain is greater than that of the others. There is no direct evidence for photodepolymerization of PI, but it would be possible to argue that the anisotropic photodepolymerization of PI by linearly polarized DUV irradiation caused homogeneous monodomain alignment of nematic liquid crystals. 


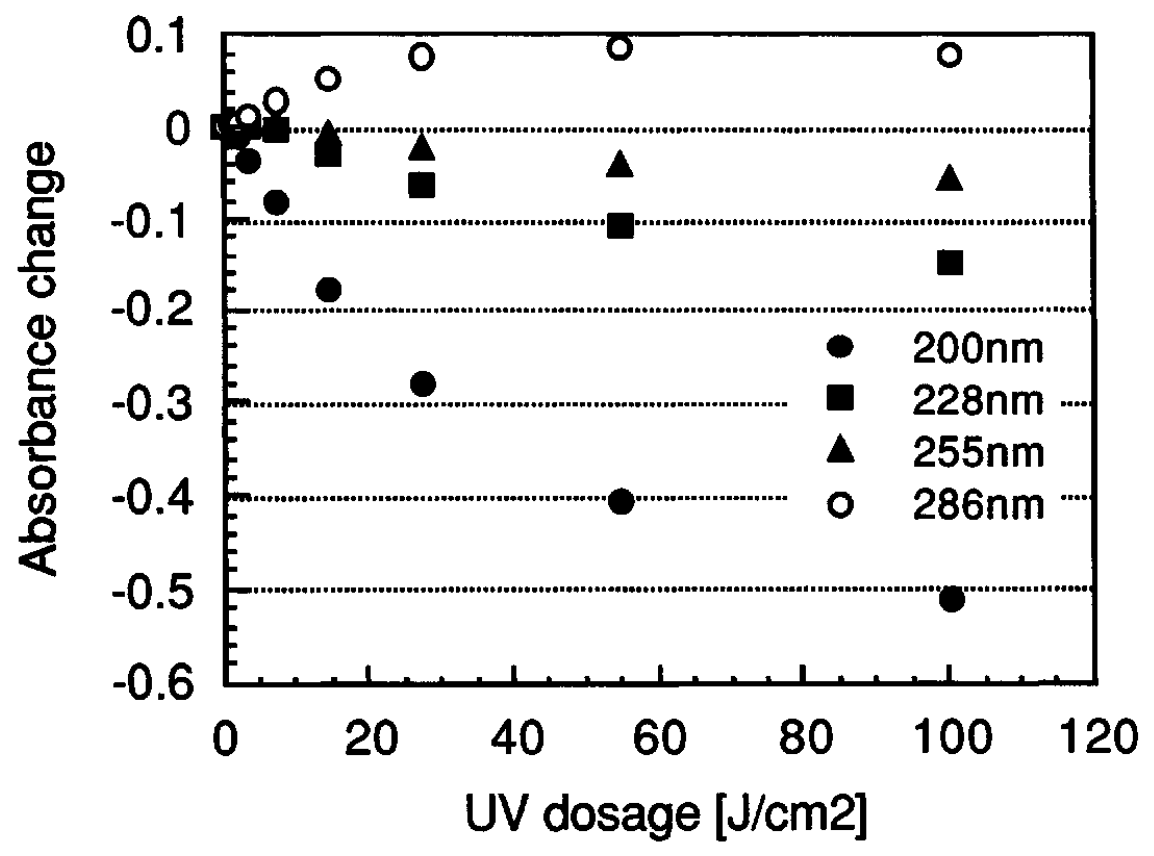

Fig. 7 Absorbance change in each peak

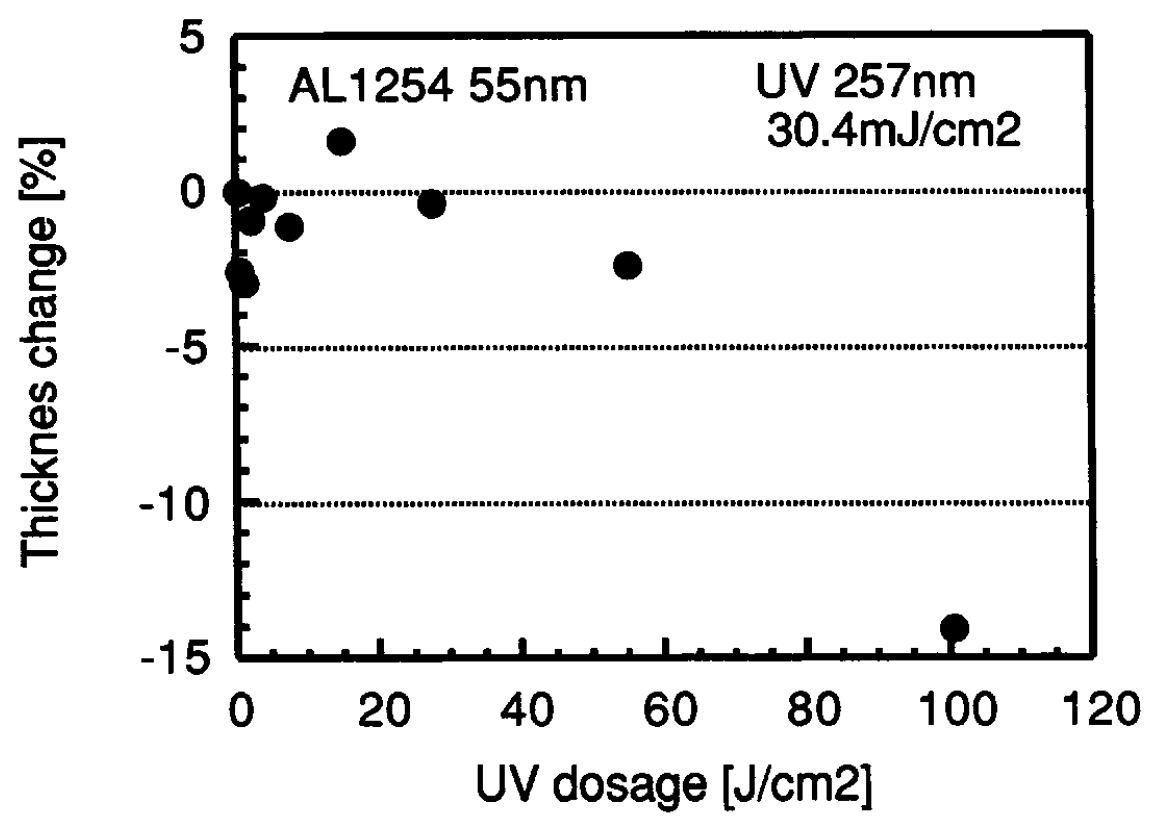

Fig. 8 Change in thickness of a PI film 


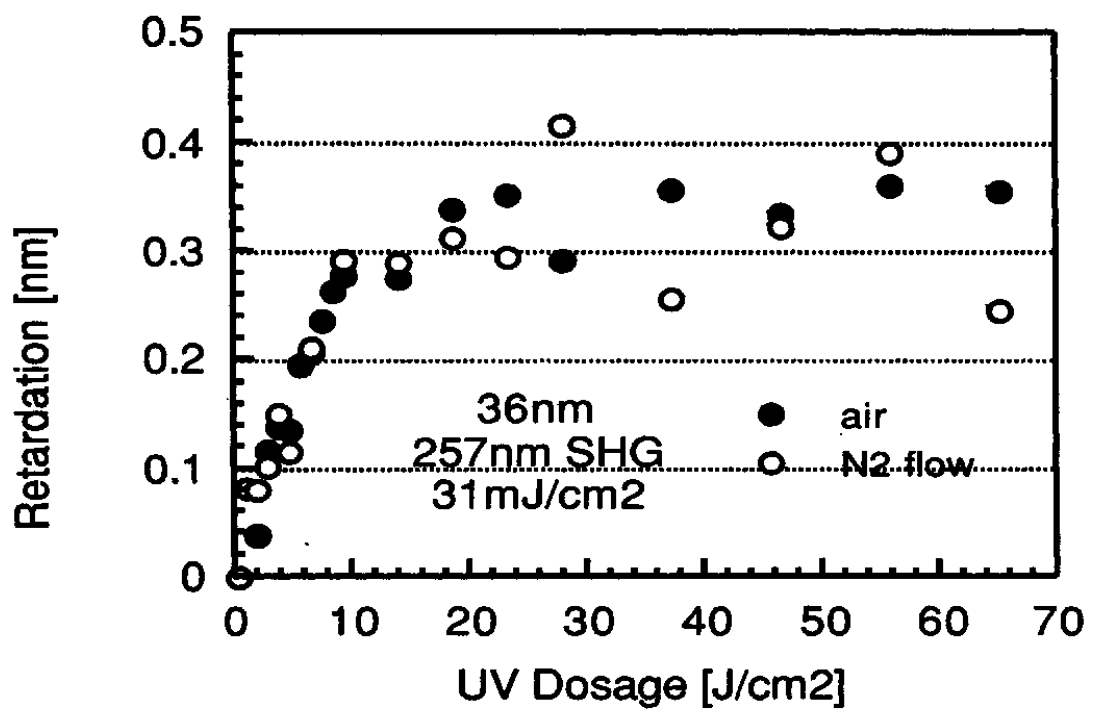

Fig. 9 Change in retardation of a PI film

\section{Summary}

We induced nematic monodomain homogeneous alignment by exposing positive-type PI to linearly polarized DUV light. The alignment direction was perpendicular to the electric field of the DUV, and its condition suddenly changed at $7 \mathrm{~J} / \mathrm{cm}^{2}$. We explained this phenomenon as resulting from a new mechanism, anisotropic photodepolymerization of PI.

\section{References}

1. W. M. Gibbons, P. J. Shannon, S. Sun, and B. J. Swetlin, "Surface-mediated alignment of nematic liquid crystals with polarized laser light," Nature 351, 49 (1991)

2. Y. Jawanishi, T. Tamaki, T. Seki, M. Sakuragi, and J. Ichimura, "Nematic homogeneous alignment regulated by the polarization photochromism of surface azobenzenes," Mol. Cryst. Liq. Cryst. 218, 153 (1992)

3. M. Schadt, K. Schmitt, V. Jozinkov, and V. Chigrinov, "Surface-induced parallel alignment of liquid crystals by linearly polymerized photopolymers," Jpn. J. Appl. Phys. 31, 2155 (1992)

4. L. Kan and K. C. Kao, "Ultraviolet absorption and photoconduction spectra of polyimide films fabricated at various curing temperatures," J. Chem. Phys. 98, 3445 (1993)

5. P. G. de Gennes, The Physics of Liquid Crystals, Clarendon, Oxford (1974) 